\title{
Role of Serotonin Neurons in L-DOPA- and Graft-Induced Dyskinesia in a Rat Model of Parkinson's Disease
}

\author{
Eunju Shin, ${ }^{1}$ Elisabetta Tronci, ${ }^{2}$ and Manolo Carta ${ }^{2}$ \\ ${ }^{1}$ Division of Neurobiology, Wallenberg Neuroscience Center, Lund University, 22184 Lund, Sweden \\ ${ }^{2}$ Department of Biomedical Science, Cagliari University, Cittadella Universitaria, SS $554 \mathrm{~km}$ 4.500, 09042 Monserrato, Italy
}

Correspondence should be addressed to Manolo Carta, manolocarta@unica.it

Received 16 February 2012; Revised 2 April 2012; Accepted 10 April 2012

Academic Editor: Gilberto Fisone

Copyright ( 2012 Eunju Shin et al. This is an open access article distributed under the Creative Commons Attribution License, which permits unrestricted use, distribution, and reproduction in any medium, provided the original work is properly cited.

\begin{abstract}
L-DOPA, the most effective drug to treat motor symptoms of Parkinson's disease, causes abnormal involuntary movements, limiting its use in advanced stages of the disease. An increasing body of evidence points to the serotonin system as a key player in the appearance of L-DOPA-induced dyskinesia (LID). In fact, exogenously administered L-DOPA can be taken up by serotonin neurons, converted to dopamine and released as a false transmitter, contributing to pulsatile stimulation of striatal dopamine receptors. Accordingly, destruction of serotonin fibers or silencing serotonin neurons by serotonin agonists could counteract LID in animal models. Recent clinical work has also shown that serotonin neurons are present in the caudate/putamen of patients grafted with embryonic ventral mesencephalic cells, producing intense serotonin hyperinnervation. These patients experience graftinduced dyskinesia (GID), a type of dyskinesia phenotypically similar to the one induced by L-DOPA but independent from its administration. Interestingly, the $5-\mathrm{HT}_{1 \mathrm{~A}}$ receptor agonist buspirone has been shown to suppress GID in these patients, suggesting that serotonin neurons might be involved in the etiology of GID as for LID. In this paper we will discuss the experimental and clinical evidence supporting the involvement of the serotonin system in both LID and GID.
\end{abstract}

\section{Introduction}

Parkinson's disease (PD) is the second most common neurodegenerative disease and is characterized by loss of dopamine (DA) neurons in the substantia nigra. The cell loss results in decreased activation of striatal DA receptors, thus causing motor impairments, such as tremor, rigidity, bradykinesia, and postural instability. The DA precursor L-3,4-dihydroxyphenylalanine (L-DOPA) represents the most effective drug to alleviate the motor symptoms. Although this medication is very efficient during the first few years of administration, its efficacy gradually diminishes overtime, and uncontrolled excessive movements, known as dyskinesia, appear as a side effect after a variable number of years in most of patients, limiting the use of L-DOPA in advanced stages of the disease.

A better understanding of the mechanisms underlying the appearance of dyskinesia has been achieved during recent years using animal models of L-DOPA-induced dyskinesia (LID). In fact, abnormal involuntary movements (AIMs) develop in response to sub-chronic L-DOPA treatment in 6-hydroxydopamine (6-OHDA)-lesioned rats and 1-methyl4-phenyl-1,2,3,6-tetrahydropyridine (MPTP)-treated monkeys, resembling peak-dose dyskinesia seen in patients [14]. Using these models, a number of alterations have been identified at the level of striatal neurons of dyskinetic subjects, such as abnormal trafficking of $\mathrm{DA}_{1}$ and $N$-methylD-aspartate (NMDA) receptors $[5,6]$, leading to alterations in key striatal signaling pathways.

More recently, the serotonin system has emerged as a putative player in the induction of fluctuations in synaptic DA levels following administration of L-DOPA in animal models of PD, which cause pulsatile stimulation of DA receptors and promote the maladaptive changes that characterize the parkinsonian dyskinetic brain [7-11]. The emerging role of serotonin neurons in LID has also prompted researchers to investigate a possible involvement of these neurons in the appearance of off-drug dyskinesia that has emerged in a subset of PD patients following transplantation of fetal 
ventral mesencephalic DA neuroblasts, namely, graftinduced dyskinesia (GID) $[12,13]$. In this paper, we will discuss the recent experimental and clinical evidence supporting a role of serotonin neurons both in LID and GID.

\section{The Role of Serotonin Neurons in the Induction of L-DOPA-Induced Dyskinesia}

Progression of DA neuron degeneration represents the first risk factor for development of LID in patients. In fact, the efficacy of L-DOPA in providing its therapeutic effect during the first years of administration is conceivably due to the ability of spared DA neurons to take up exogenously administered L-DOPA, convert it to DA, and release it into the synaptic cleft, but also to regulate synaptic DA levels via the $\mathrm{D}_{2}$ autoreceptor and DA transporter (DAT). When the disease is in its early stage, sufficient DA terminals remain in the striatum to efficiently mediate a feedback-controlled mechanism of release. The ability of the spared DA terminals to prevent development of AIMs upon L-DOPA treatment is well demonstrated in a recent report by Ulusoy et al. [14]. In this study, a significant DA deficiency was established in rats by viral vector delivery of short hairpin RNA for the tyrosine hydroxylase (TH) enzyme, without affecting cell survival. Animals were then made dyskinetic by subchronic treatment with the direct DA agonist apomorphine; however, when treated with L-DOPA, the same rats appeared to be fully resistant to the induction of LID, despite apomorphine treatment had already promoted postsynaptic alterations, such as increased striatal FosB expression.

The ability of the presynaptic DA compartment to prevent excessive DA receptor stimulation, and aberrant downstream signaling, even in presence of supersensitive striatal DA receptors is also confirmed in transplantation studies. In fact, ventral mesencephalic DA graft, which reconstitutes the presynaptic buffering capacity into the lesioned and maladapted striatum, normalizes the response of L-DOPAprimed dyskinetic rats to L-DOPA administration [7]. In light with these results, it is conceivable to think that the efficacy of L-DOPA during the first years of administration is also due to the presence of a sufficient number of DA neurons that can buffer the exogenously administered L-DOPA, and provide a source of regulated DA release. However, with the progression of DA degeneration, this buffering capacity, and thus the ability to provide physiological level of DA receptor stimulation, is progressively lost. In this situation, the serotonin neurons come to play a major role in L-DOPAderived DA production and release, as they possess both the aromatic amino acid decarboxylase enzyme (AADC) and the vesicular monoamine transporter (VMAT). Unlike DA neurons, though, serotonin neurons cannot regulate the extracellular levels of DA due to the lack of the autoregulatory loop, hence, causing un-controlled DA release following L-DOPA administration. DA released from serotonin neurons will therefore act in concert with the intermittent nature of the orally administered L-DOPA to cause pulsatile stimulation of DA receptors, and thus, changes in downstream signaling pathways at striatal neurons. In support of this view, removal of serotonin innervation by toxin lesion was shown to produce about $80 \%$ reduction in L-DOPA-derived striatal extracellular DA levels [15], and to induce a nearto-complete suppression of LID in dyskinetic rats $[8,16]$. Accordingly, pharmacological silencing of serotonin neuron activity by $5-\mathrm{HT}_{1 \mathrm{~A}}$ and $5-\mathrm{HT}_{1 \mathrm{~B}}$ receptor agonists has been shown to reduce L-DOPA-derived extracellular DA levels [17], and to suppress LID in rats [8] as well as in MPTPtreated monkeys [18]. In addition, chronic administration of the $5-\mathrm{HT}_{1}$ agonists was able to prevent the development of dyskinesia and upregulation of FosB expression in the striatum of 6-OHDA-lesioned rats [18], thus linking dysregulated DA release from serotonin terminals with the induction of a well-known striatal marker of dyskinesia. Interestingly, simultaneous activation of $5-\mathrm{HT}_{1 \mathrm{~A}}$ and $5-\mathrm{HT}_{1 \mathrm{~B}}$ receptors was found to trigger a potent synergistic effect in the suppression of LID in both rats and monkeys $[8,18]$. In fact, LID was nearly fully abolished at doses of combined agonists that were ineffective when given individually. This finding has now led to the initiation of a first double-blind, proof-of-concept clinical trial employing a mixed $5-\mathrm{HT}_{1 \mathrm{~A} / 1 \mathrm{~B}}$ receptor agonist in dyskinetic patients.

In confirming the interaction between exogenously administered L-DOPA and serotonin neurons, Navailles and coworkers have recently demonstrated that serotonin neuron-dependent DA release takes place, upon chronic L-DOPA treatment in 6-OHDA-lesioned rats, not only in the striatum but also in other brain areas receiving sufficient serotonin innervation, such as substantia nigra, hippocampus, and prefrontal cortex [19]. Moreover, L-DOPA administration appears to result in reduced striatal serotonin tissue content in 6-OHDA-lesioned rats [8]. The latter result supports the existence of a competition between serotonin and DA for serotonergic vesicles, which causes serotonin depletion. Thus, an increasing body of experimental evidence points to the serotonin system as a key player in the appearance of LID.

Progressive reduction of L-DOPA-derived extracellular DA levels upon chronic L-DOPA treatment has been recently found in 6-OHDA-lesioned rats [20, 21], leading some researcher to question the role of serotonin neurons in the appearance of LID [21]. Nevertheless, we believe that the potent inhibitory effect of 5,7-dihydroxytryptamine (5,7DHT) lesion on both development and expression of dyskinesia in 6-OHDA-lesioned rats [8], together with the striking suppression of LID induced by low doses of $5-\mathrm{HT}_{1 \mathrm{~A}}+5-$ $\mathrm{HT}_{1 \mathrm{~B}}$ receptor agonists both in rats and macaques [18] provided unquestionable evidence supporting an important role of serotonin neurons, at least in animal models of PD. It should be taken into account that during chronic administration of L-DOPA, postsynaptic DA receptors become supersensitive; thus, the dyskinetic response to L-DOPA might be maintained by lower extracellular DA levels once postsynaptic alterations have been already induced. The relevance for the human disease of the progressive reduction of extracellular DA levels seen upon chronic L-DOPA in the rat 6-OHDA model remains to be established, as L-DOPAderived synaptic DA levels were shown to increase with progression of the disease in a positron emission tomography (PET) imaging study in PD patients [22]. 
Interestingly, Rylander and coworkers have recently shown marked serotonin hyperinnervation in the lesioned striatum of parkinsonian dyskinetic subjects across different species, including patients, thus raising the possibility that serotonin neurons play a relevant role in the emergence of LID also in the patients $[10,11,23]$. This study suggested that L-DOPA treatment may be able to provoke sprouting of serotonin axon terminals and change their morphology, hence, possibly enhancing the fluctuations in extracellular DA concentration, consistent with findings of de la Fuente-Fernandez and coworkers in their PET imaging study $[22,24]$.

In further experimental support for a detrimental effect of serotonin neurons on LID, grafted serotonin neurons, which induced an intense hyperinnervation of the grafted striatum, exacerbated LID in both partial and complete DA lesioned rats $[7,25]$, raising the possibility that inclusion of these cells in the grafted tissue may have detrimental effects on LID in grafted PD patients.

\section{The Role of Serotonin Neurons in the Modulation of Graft-Induced Dyskinesia}

Transplantation of fetal ventral mesencephalic neurons is a therapeutic approach to PD that has already been tested in clinical trials [26-30]. While the variability in the outcome of these studies halted further investigations, the presence of highly responsive patients provided proof-of-concept that this therapeutic intervention can be significantly beneficial. Indeed, there is now general agreement that the reasons accounting for the observed variability rely on the lack of standardization of the cell preparations, surgical procedures, as well as on the selection of patients and presence or absence of postsurgical immunosuppressive treatment [31]. However, another element that has contributed to raise concern about fetal transplantation is the appearance in a subset of grafted patients of off-drug dyskinesia [29, 31-33], a form of involuntary movements that is phenotypically similar to the one induced by L-DOPA but independent from its administration. The recent findings on the role of serotonin neurons in the induction of LID has led to hypothesize that serotonin neurons included in the graft may also play a role in GID $[8,16,18]$.

In fact, serotonin neuroblasts are located in close vicinity of the DA ones in the fetal ventral mesencephalic area that is dissected for transplantation. Accordingly, about $50 \%$ of grafted cells were found to be serotonin neurons in a postmortem analysis of the caudate-putamen of grafted patients [34].

In possible support of this hypothesis, in a recent PET study, Politis and colleagues have found intense serotonergic hyperinnervation in the striatum of grafted patients showing GID [12, 13]. Interestingly, administration of the partial $5-\mathrm{HT}_{1 \mathrm{~A}}$ agonist buspirone suppressed GID in all tested patients. An involvement of the serotonin neurons in GID is further supported by the high serotonin transporter (SERT)/ DAT ratio found in one GID patient compared to both healthy age-matched control and non-grafted PD patients [12]. Thus, it is postulated that serotonin terminals may take up DA released by the graft through SERT, and release DA, as a false transmitter, away from the uptake site, in striatal regions lacking sufficient DA innervation, thus, leading to activation of supersensitive DA receptors. However, it should be acknowledged that these clinical observations have been made in a very few subjects, and further evidence should be provided. In particular, it would be important to investigate the state of the striatal serotonin innervation also in patients free of GID.

Although spontaneous GID, that is dyskinesia in the absence of any drug treatment, is inconsistent in grafted rodents $[35,36]$, it does appear after administration of a low dose of amphetamine [35, 37], which is known to evoke massive DA release from grafted DA neurons [38]. These abnormal movements can be scored with the same scale used for LID [35, 37], and are now widely used as a convenient and reproducible model of GID [7, 39-42]. While appearance of GID in this rat model is clearly dependent on the presence of an adequate number of DA neurons in the graft, we have recently found a bidirectional modulatory effect of endogenous serotonin neurons on GID. In fact, reduction of serotonin neuron activity, by a combination of $5-\mathrm{HT}_{1 \mathrm{~A}}$ and $5-\mathrm{HT}_{1 \mathrm{~B}}$ receptor agonists, produced a significant reduction of GID, while increased serotonin neuron release by fenfluramine exacerbated GID [43]. Strikingly, administration of a low dose of buspirone $(1 \mathrm{mg} / \mathrm{kg})$ completely suppressed GID, as seen in grafted patients. Interestingly, removal of the endogenous serotonin innervation by specific toxin lesions appeared to abolish the anti-GID effect of the selective $5-\mathrm{HT}_{1}$ agonists, both in serotonin-containing and serotonin-free grafts, suggesting that the modulatory effect on GID may be due to the endogenous rather than the graft-derived serotonin neurons, at least in our experimental conditions. By contrast, neither removal of the endogenous serotonin innervation nor pretreatment with the selective $5-\mathrm{HT}_{1 \mathrm{~A}}$ antagonist WAY100135 reduced the anti-GID efficacy of buspirone. In fact, buspirone is also known to possess antagonistic properties on $\mathrm{D}_{2}$ receptors [44-47]. In support for a $\mathrm{D}_{2}$-mediated effect of buspirone, similar anti-GID effect was induced by a low dose of the selective $\mathrm{D}_{2}$ receptor antagonist eticlopride $(0.03 \mathrm{mg} / \mathrm{kg})$. Thus, our data support a modulatory role of the endogenous serotonin neurons on expression of GID, as well as a peculiar role of $\mathrm{D}_{2}$ receptors. Indeed, both buspirone and eticlopride were ineffective against LID at doses fully suppressing GID [43].

Further work is required to understand whether inclusion of serotonin neurons in the graft can be detrimental for appearance of GID, although current experimental data do not support this hypothesis.

\section{Conclusion}

Overall, loss of DA in basal ganglia circuits and DA replacement by chronic L-DOPA administration result in complex alterations in the parkinsonian brain, that affect several systems and key signaling proteins, most of which remain poorly understood. DA released as a false transmitter from serotonin neurons appears to play a key role in initiating these events, at least in animal models. Serotonin neurons, 
therefore, represent an intriguing pharmacological target to treat already established LID and/or to prevent the events leading to the appearance of LID from taking place. Recent evidence suggests that serotonin neurons may also participate to the induction of dyskinesia seen in the off-state in grafted PD patients. The upcoming new clinical trial, funded by the European Community, employing fetal ventral mesencephalic cells will answer the question whether optimization of the surgical procedures and preparation of the grafted material, including exclusion of serotonin neuroblasts, will improve clinical outcome and avoid appearance of GID.

\section{Acknowledgments}

E. Shin is supported by the Strategic Research Area Multipark at Lund University and E. Tronci is supported by Regione Autonoma delle Sardegna within the Master\&Back Program at Cagliari University.

\section{References}

[1] E. Bézard, S. Ferry, U. Mach et al., "Attenuation of levodopainduced dyskinesia by normalizing dopamine D3 receptor function," Nature Medicine, vol. 9, no. 6, pp. 762-767, 2003.

[2] M. P. Hill, E. Bézard, S. G. McGuire et al., "Novel antiepileptic drug levetiracetam decreases dyskinesia elicited by L-dopa and ropinirole in the MPTP-lesioned marmoset," Movement Disorders, vol. 18, no. 11, pp. 1301-1305, 2003.

[3] A. Hsu, D. M. Togasaki, E. Bézard et al., "Effect of the D3 dopamine receptor partial agonist BP897 [N-[4-(4-(2-methoxyphenyl)piperazinyl)butyl]-2-naphthamide] on L-3,4dihydroxyphenylalanine-induced dyskinesias and parkinsonism in squirrel monkeys," Journal of Pharmacology and Experimental Therapeutics, vol. 311, no. 2, pp. 770-777, 2004.

[4] M. Lundblad, M. Andersson, C. Winkler, D. Kirik, N. Wierup, and M. A. Cenci, "Pharmacological validation of behavioural measures of akinesia and dyskinesia in a rat model of Parkinson's disease," European Journal of Neuroscience, vol. 15, no. 1, pp. 120-132, 2002.

[5] A. Berthet, G. Porras, E. Doudnikoff et al., "Pharmacological analysis demonstrates dramatic alteration of $\mathrm{D}_{1}$ dopamine receptor neuronal distribution in the rat analog of L-DOPAinduced dyskinesia," Journal of Neuroscience, vol. 29, no. 15, pp. 4829-4835, 2009.

[6] C. Guigoni, E. Doudnikoff, Q. Li, B. Bloch, and E. Bézard, "Altered $\mathrm{D}_{1}$ dopamine receptor trafficking in parkinsonian and dyskinetic non-human primates," Neurobiology of Disease, vol. 26, no. 2, pp. 452-463, 2007.

[7] T. Carlsson, M. Carta, C. Winkler, A. Björklund, and D. Kirik, "Serotonin neuron transplants exacerbate L-DOPA-induced dyskinesias in a rat model of Parkinson's disease," Journal of Neuroscience, vol. 27, no. 30, pp. 8011-8022, 2007.

[8] M. Carta, T. Carlsson, D. Kirik, and A. Björklund, "Dopamine released from 5-HT terminals is the cause of L-DOPA-induced dyskinesia in parkinsonian rats," Brain, vol. 130, no. 7, pp. 1819-1833, 2007.

[9] A. Muñoz, T. Carlsson, E. Tronci, D. Kirik, A. Björklund, and M. Carta, "Serotonin neuron-dependent and -independent reduction of dyskinesia by $5-\mathrm{HT}_{1 \mathrm{~A}}$ and $5-\mathrm{HT}_{1 \mathrm{~B}}$ receptor agonists in the rat Parkinson model," Experimental Neurology, vol. 219, no. 1, pp. 298-307, 2009.
[10] D. Rylander, M. Parent, S. S. O-Sullivan et al., "Maladaptive plasticity of serotonin axon terminals in levodopa-induced dyskinesia," Annals of Neurology, vol. 68, no. 5, pp. 619-628, 2010.

[11] B. Y. Zeng, M. M. Iravani, M. J. Jackson, S. Rose, A. Parent, and P. Jenner, "Morphological changes in serotoninergic neurites in the striatum and globus pallidus in levodopa primed MPTP treated common marmosets with dyskinesia," Neurobiology of Disease, vol. 40, no. 3, pp. 599-607, 2010.

[12] M. Politis, W. H. Oertel, K. Wu et al., "Graft-induced dyskinesias in Parkinson's disease: high striatal serotonin/dopamine transporter ratio," Movement Disorders, vol. 26, no. 11, pp. 1997-2003, 2011.

[13] M. Politis, K. Wu, C. Loane et al., "Serotonergic neurons mediate dyskinesia side effects in Parkinson's patients with neural transplants," Science Translational Medicine, vol. 2, no. 38, Article ID 38ra46, 2010.

[14] A. Ulusoy, G. Sahin, and D. Kirik, "Presynaptic dopaminergic compartment determines the susceptibility to L-DOPAinduced dyskinesia in rats," Proceedings of the National Academy of Sciences of the United States of America, vol. 107, no. 29, pp. 13159-13164, 2010.

[15] H. Tanaka, K. Kannari, T. Maeda, M. Tomiyama, T. Suda, and M. Matsunaga, "Role of serotonergic neuron in L-DOPAderived extracellular dopamine in the striatum of 6-OHDAlesioned rats," NeuroReport, vol. 10, no. 3, pp. 631-634, 1999.

[16] K. L. Eskow, K. B. Dupre, C. J. Barnum, S. O. Dickinson, J. Y. Park, and C. Bishop, "The role of the dorsal raphe nucleus in the development, expression, and treatment of L-dopainduced dyskinesia in hemiparkinsonian rats," Synapse, vol. 63, no. 7, pp. 610-620, 2009.

[17] H. S. Lindgren, D. R. Andersson, S. Lagerkvist, H. Nissbrandt, and M. A. Cenci, "L-DOPA-induced dopamine efflux in the striatum and the substantia nigra in a rat model of Parkinson's disease: temporal and quantitative relationship to the expression of dyskinesia," Journal of Neurochemistry, vol. 112, no. 6, pp. 1465-1476, 2010.

[18] A. Muñoz, Q. Li, F. Gardoni et al., "Combined 5- $\mathrm{HT}_{1 \mathrm{~A}}$ and 5$\mathrm{HT}_{1 \mathrm{~B}}$ receptor agonists for the treatment of L-DOPA-induced dyskinesia," Brain, vol. 131, no. 12, pp. 3380-3394, 2008.

[19] S. Navailles, A. Benazzouz, B. Bioulac, C. Gross, and P. De Deurwaerdère, "High-frequency stimulation of the subthalamic nucleus and L-3,4-dihydroxyphenylalanine inhibit in vivo serotonin release in the prefrontal cortex and hippocampus in a rat model of Parkinson's disease," Journal of Neuroscience, vol. 30, no. 6, pp. 2356-2364, 2010.

[20] S. Navailles, B. Bioulac, C. Gross, and P. De Deurwaerdère, "Chronic L-DOPA therapy alters central serotonergic function and L-DOPA-induced dopamine release in a regiondependent manner in a rat model of Parkinson's disease," Neurobiology of Disease, vol. 41, no. 2, pp. 585-590, 2011.

[21] N. Nevalainen, S. Af Bjerken, M. Lundblad, G. A. Gerhardt, and I. Stromberg, "Dopamine release from serotonergic nerve fibers is reduced in L-DOPA-induced dyskinesia," Journal of Neurochemistry, vol. 118, pp. 12-23, 2011.

[22] R. de La Fuente-Fernández, V. Sossi, Z. Huang et al., "Levodopa-induced changes in synaptic dopamine levels increase with progression of Parkinson's disease: implications for dyskinesias," Brain, vol. 127, no. 12, pp. 2747-2754, 2004.

[23] S. Gil, C. Park, J. Lee, and H. Koh, "The roles of striatal serotonin and 1-amino-acid decarboxylase on 1-DOPA-induced dyskinesia in a hemiparkinsonian rat model," Cellular and Molecular Neurobiology, vol. 30, no. 6, pp. 817-825, 2010. 
[24] R. de La Fuente-Fernández, M. Schulzer, E. Mak, D. B. Calne, and A. J. Stoessl, "Presynaptic mechanisms of motor fluctuations in Parkinson's disease: a probabilistic model," Brain, vol. 127, no. 4, pp. 888-899, 2004.

[25] T. Carlsson, M. Carta, A. Muñoz et al., "Impact of grafted serotonin and dopamine neurons on development of LDOPA-induced dyskinesias in parkinsonian rats is determined by the extent of dopamine neuron degeneration," Brain, vol. 132, no. 2, pp. 319-335, 2009.

[26] C. R. Freed, R. E. Breeze, N. L. Rosenberg et al., "Survival of implanted fetal dopamine cells and neurologic improvement 12 to 46 months after transplantation for Parkinson's disease," The New England Journal of Medicine, vol. 327, no. 22, pp. 1549-1555, 1992.

[27] O. Lindvall, G. Sawle, H. Widner et al., "Evidence for longterm survival and function of dopaminergic grafts in progressive Parkinson's disease," Annals of Neurology, vol. 35, no. 2, pp. 172-180, 1994.

[28] O. Lindvall, H. Widner, S. Rehncrona et al., "Transplantation of fetal dopamine neurons in Parkinson's disease: one-year clinical and neurophysiological observations in two patients with putaminal implants," Annals of Neurology, vol. 31, no. 2, pp. 155-165, 1992.

[29] C. W. Olanow, C. G. Goetz, J. H. Kordower et al., "A doubleblind controlled trial of bilateral fetal nigral transplantation in Parkinson's disease," Annals of Neurology, vol. 54, no. 3, pp. 403-414, 2003.

[30] P. Piccini, D. J. Brooks, A. Björklund et al., "Dopamine release from nigral transplants visualized in vivo in a Parkinson's patient," Nature Neuroscience, vol. 2, no. 12, pp. 1137-1140, 1999.

[31] E. L. Lane, A. Björklund, S. B. Dunnett, and C. Winkler, "Neural grafting in Parkinson's disease. Unraveling the mechanisms underlying graft-induced dyskinesia," Progress in Brain Research, vol. 184, pp. 295-309, 2010.

[32] C. R. Freed, P. E. Greene, R. E. Breeze et al., “Transplantation of embryonic dopamine neurons for severe Parkinson's disease," The New England Journal of Medicine, vol. 344, no. 10, pp. 710-719, 2001.

[33] P. Hagell, P. Piccini, A. Björklund et al., "Dyskinesias following neural transplantation in Parkinson's disease," Nature Neuroscience, vol. 5, no. 7, pp. 627-628, 2002.

[34] I. Mendez, A. Vĩuela, A. Astradsson et al., "Dopamine neurons implanted into people with Parkinson's disease survive without pathology for 14 years," Nature Medicine, vol. 14, no. 5, pp. 507-509, 2008.

[35] E. L. Lane, C. Winkler, P. Brundin, and M. A. Cenci, "The impact of graft size on the development of dyskinesia following intrastriatal grafting of embryonic dopamine neurons in the rat," Neurobiology of Disease, vol. 22, no. 2, pp. 334-345, 2006.

[36] A. Vinuela, P. J. Hallett, C. Reske-Nielsen et al., "Implanted reuptake-deficient or wild-type dopaminergic neurons improve on L-dopa dyskinesias without OFF-dyskinesias in a rat model of Parkinson's disease," Brain, vol. 131, no. 12, pp. 3361-3379, 2008.

[37] T. Carlsson, C. Winkler, M. Lundblad, M. A. Cenci, A. Björklund, and D. Kirik, "Graft placement and uneven pattern of reinnervation in the striatum is important for development of graft-induced dyskinesia," Neurobiology of Disease, vol. 21, no. 3, pp. 657-668, 2006.

[38] T. Zetterstrom, M. Herrera-Marschitz, and U. Ungerstedt, "Simultaneous measurement of dopamine release and rotational behaviour in 6-hydroxydopamine denervated rats using intracerebral dialysis," Brain Research, vol. 376, no. 1, pp. 1-7, 1986.

[39] E. L. Lane, L. Vercammen, M. A. Cenci, and P. Brundin, "Priming for L-DOPA-induced abnormal involuntary movements increases the severity of amphetamine-induced dyskinesia in grafted rats," Experimental Neurology, vol. 219, no. 1, pp. 355358, 2009.

[40] E. L. Lane, D. Soulet, L. Vercammen, M. A. Cenci, and P. Brundin, "Neuroinflammation in the generation of posttransplantation dyskinesia in Parkinson's disease," Neurobiology of Disease, vol. 32, no. 2, pp. 220-228, 2008.

[41] E. L. Lane, P. Brundin, and M. A. Cenci, "Amphetamineinduced abnormal movements occur independently of both transplant- and host-derived serotonin innervation following neural grafting in a rat model of Parkinson's disease," Neurobiology of Disease, vol. 35, no. 1, pp. 42-51, 2009.

[42] J. Garcia, T. Carlsson, M. Döbrössy, G. Nikkhah, and C. Winkler, "Extent of pre-operative L-DOPA-induced dyskinesia predicts the severity of graft-induced dyskinesia after fetal dopamine cell transplantation," Experimental Neurology, vol. 232, pp. 270-279, 2011.

[43] E. Shin, J. Garcia, C. Winkler, A. Björklund, and M. Carta, "Serotonergic and dopaminergic mechanisms in graftinduced dyskinesia in a rat model of Parkinson's disease," Neurobiology of Disease. In press.

[44] A. S. Eison and D. L. Temple, "Buspirone: review of its pharmacology and current perspectives on its mechanism of action," American Journal of Medicine, vol. 80, no. 3, pp. 1-9, 1986.

[45] B. A. McMillen, R. T. Matthews, and M. K. Sanghera, "Dopamine receptor antagonism by the novel anti-anxiety drug, buspirone," Journal of Neuroscience, vol. 3, no. 4, pp. 733-738, 1983.

[46] H. J. Rijnders and J. L. Slangen, "The discriminative stimulus properties of buspirone involve dopamine-2 receptor antagonist activity," Psychopharmacology, vol. 111, no. 1, pp. 55-61, 1993.

[47] J. Scuvee-Moreau, I. Giesbers, and A. Dresse, "Electrophysiological and microiontophoretic studies with buspirone: influence on the firing rate of central monoaminergic neurons and their responsiveness to dopamine, clonidine or GABA," Archives Internationales de Physiologie et de Biochimie, vol. 95, no. 5, pp. 439-446, 1987. 


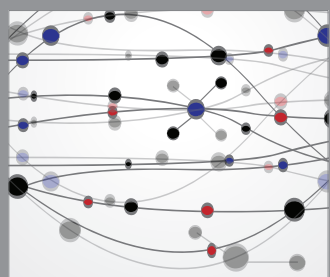

The Scientific World Journal
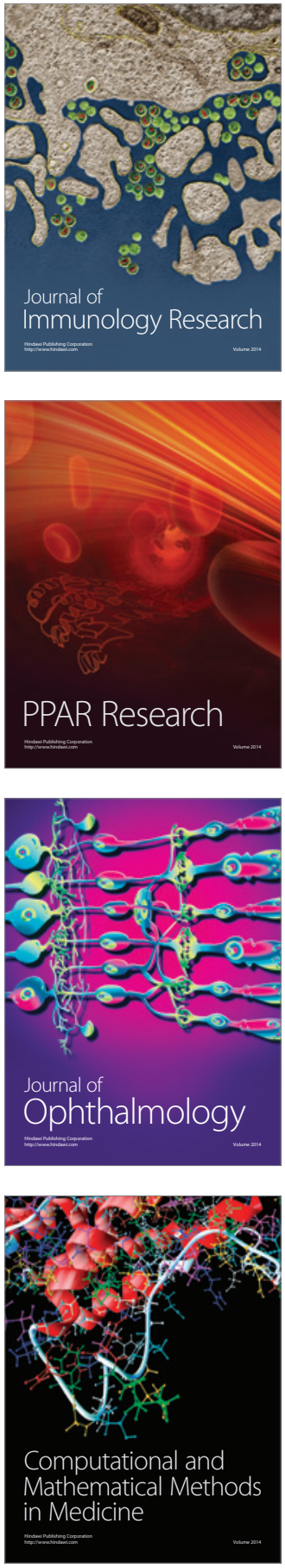

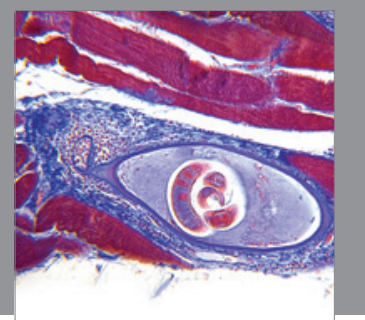

Gastroenterology

Research and Practice
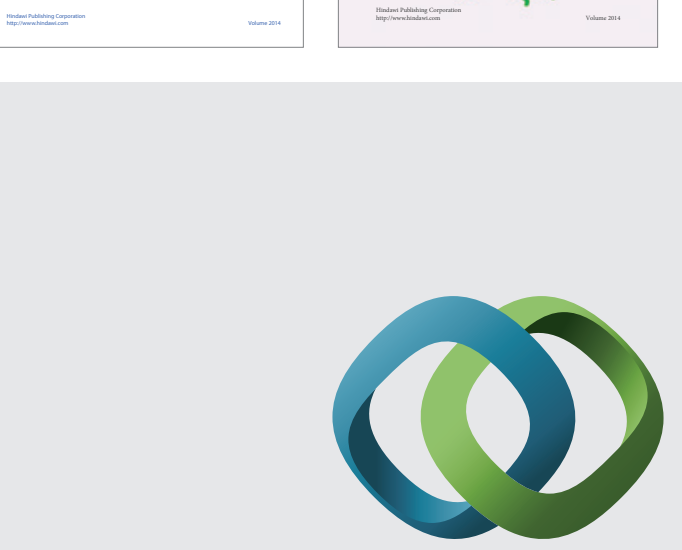

\section{Hindawi}

Submit your manuscripts at

http://www.hindawi.com
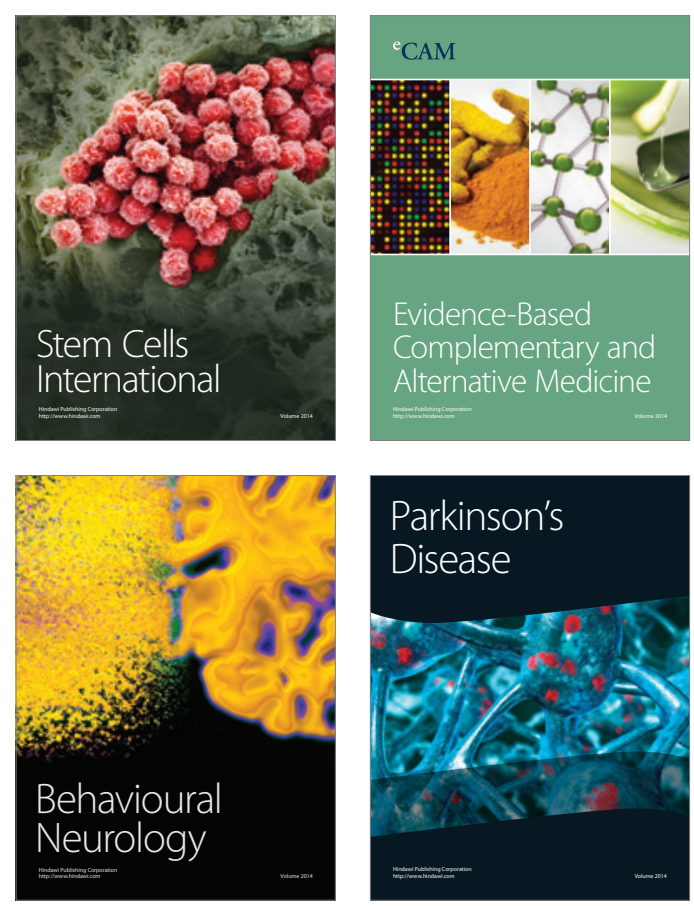

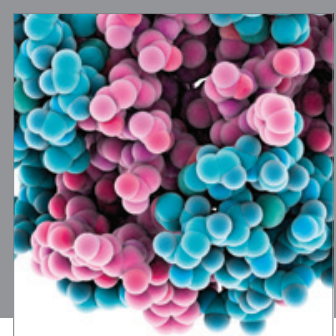

Journal of
Diabetes Research

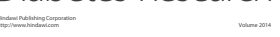

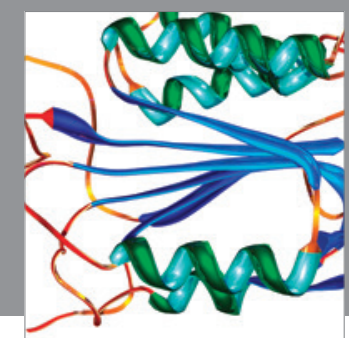

Disease Markers
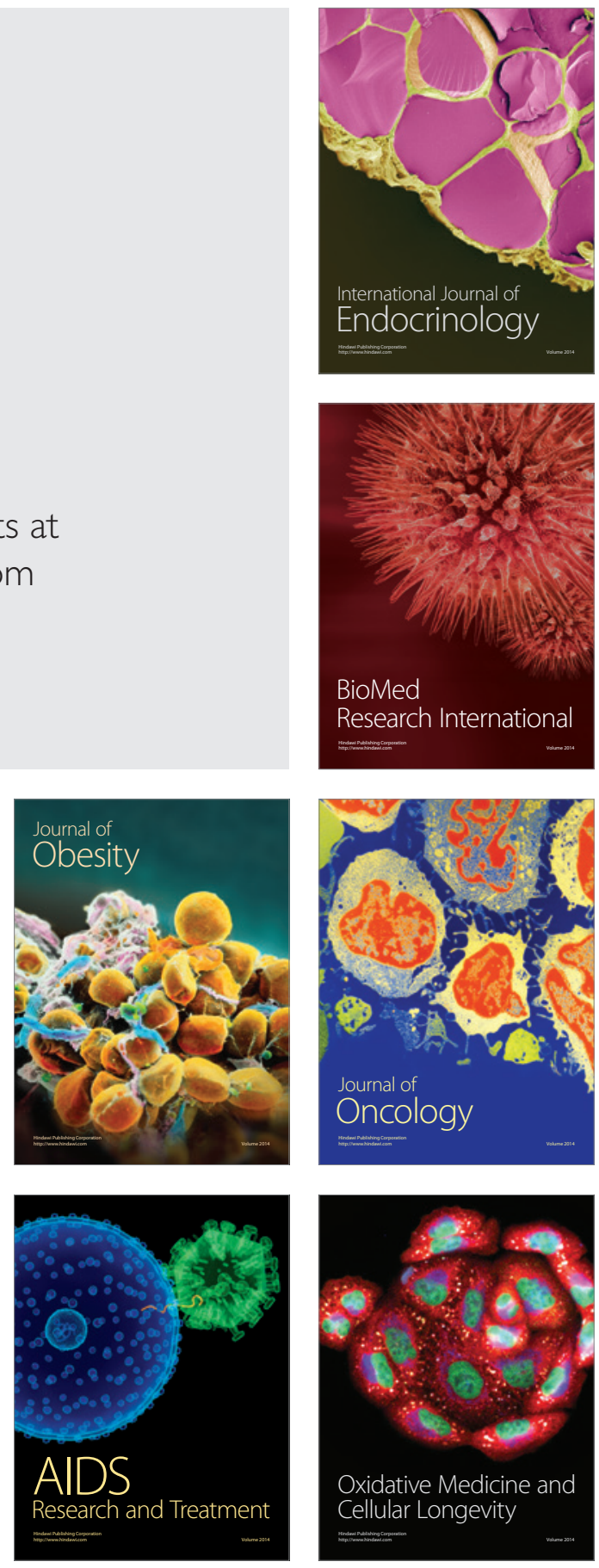